\title{
Metarhizium dendrolimatilis, a novel Metarhizium species parasitic on Dendrolimus sp. larvae
}

\author{
Chen $\mathrm{WH}^{1,2}$, Han $\mathrm{YF}^{2}$, Liang $\mathrm{JD}^{3}$, Liang $\mathrm{ZQ}^{2}$ and Jin $\mathrm{DC}^{1}$ \\ ${ }^{1}$ Institute of Entomology, College of Agriculture, Guizhou University, Guiyang, Guizhou 550025, China \\ ${ }^{2}$ Institute of Fungus Resources, College of Life Sciences, Guizhou University, Guiyang, Guizhou 550025, China \\ ${ }^{3}$ Department of Microbiology, School of Basic Medical Science, Guiyang College of Traditional Chinese Medicine, \\ Guiyang, Guizhou 550025, China
}

Chen WH, Han YF, Liang JD, Liang ZQ, Jin DC 2017 -Metarhizium dendrolimatilis, a novel Metarhizium species parasitic on Dendrolimus sp. larvae. Mycosphere 8(1), 31-37, Doi $10.5943 /$ mycosphere/8/1/4

\begin{abstract}
A novel species of the genus Meatrhizium, Metarhizium dendrolimatilis, parasitic on Dendrolimus sp. larvae, collected in Huaxi, Guiyang, Guizhou Province, China, is described based on morphological and phylogenetic evidences. This species differs morphologically from other species in the genus by its determinate synnemata, ellipsoidal conidia, and globose phialides. The phylogenetic analyses based on four loci (EF1a, RPB1, RPB2 and TUB), strongly support the novel species designation of this fungus within the Metarhizium genus, Metarhizium dendrolimatilis sp. nov.
\end{abstract}

Key words - entomopathogenic fungi - morphology - multi-gene - phylogeny

\section{Introduction}

The genus Metarhizium (Metschn.) Sorokin consists of a diverse group of asexual entomopathogenic fungi with a global distribution and a wide range of host insects. The organism has been used as bio pesticide (Roberts \& St. Leger 2004) for mite and tick control (Maniania et al. 2008, Stafford et al. 2010, Wekesa et al. 2005, 2006). Since this genus was initially established, several new species and varieties have been described based on seemingly minor morphological differences from M. anisopliae (Metschn.) Sorokīn, the type species of the genus (Latch 1965, Petch 1931, 1935). Tulloch (1976) recognized only two species, M. anisopliae and M. flavoviride W. Gams \& Rozsypal, along with a single variety M. anisopliae var. majus (J.R. Johnst.) M. C. Tulloch (as var. major) (Tulloh) in her revision of the genus. Rombach et al. (1986) segregated $M$. flavoviride W. Gams \& Rozsypal into the varieties $M$. flavoviride var. flavoviride, and by virtue of its smaller conidia, M. flavoviride var. minus Rombach, Humber \& D.W. Roberts. Liang et al. (1991) named M. taii Z.Q. Liang \& A.Y. Liu isolated from the new species, Cordyceps taii Z.Q. Liang \& A.Y. Liu, and postulated a connection between $C$. taii and M. taii based on microcyclic conidiation. Liu et al. (2001) collected a single specimen and described it as a new species, 
Cordyceps brittlebankisoides Zuo Y. Liu, Z.Q. Liang, Whalley, Y.J. Yao \& A.Y. Liu, whose anamorph (confirmed by the identical ITS sequences from the teleomorphic stroma and in vitro culture) was identified as $M$. anisopliae var. majus.

The development of molecular techniques, in particular DNA sequencing, provides valuable tools for studying phylogenetic relationships and clarifying the taxonomic position of confusing species. Single and multi-locus were used in the phylogenetic classification of Metarhizium (Bischoff et al. 2006, 2009, Curran et al. 1994, Driver et al. 2000, Rakotonirainy et al. 1994). These studies highlight the superiority of the multigene phylogenetic approaches for determination of species boundaries and relationships in Metarhizium. Kepler et al. (2014) proposed application of the name Metarhizium in a manner that approximates the concept of Metacordyceps sensu Sung et al. (2007), minus Pochonia. The newly proposed definition of Metarhizium includes green-spored species in Nomuraea, the genus Chamaeleomyces, and several species formerly included in Paecilomyces. However, no Metarhizium species have previously been found with naturally determinate synnemata (fruit body).

Recently, we screened the synnematous entomopathogenic fungi of southwest China and isolated a Metarhizium with determinate synnemata infecting a Dendrolimus larvae. Based on a combined of the morphological characters and our phylogenetic analysis, we concluded that the strain GZAC IFR1006 represents a new species and describe it here as Metarhizium dendrolimatilis.

\section{Materials \& Methods}

\section{Field collections}

A novel specimen HXDX.1006 of nomuraea-like structure was collected in the Tongmuling, Huaxi, Guiyang, China during a survey of the divergence of synnematous entomogenous fungi. The collected material was returned to the laboratory in plastic bags and stored in a refrigerator until used for microscopic examination and molecular phylogenic analysis. Strain GZAC IFR1006 was isolated from the specimen HXDX.1006 on improved PDA (1\% w/v peptone). The specimen and the strain were deposited in the Institute of Fungus Resources, Guizhou University, formally Herbarium of Guizhou Agricultural College (code, GZAC), Guiyang City, Guizhou, China.

\section{Morphological examinations}

Specimen HXDX.1006 was prepared for microscopic observation using a small amount of synnemata mounted in lactic acid-phenol-glycerin solution. The strain GZAC IFR1006, was incubated on Sabouraud's dextrose and potato dextrose agars at $25{ }^{\circ} \mathrm{C}$ for $14 \mathrm{~d}$. Observations of microscopic features were made using the Motic B series microscope fitted with a Motic Digital Moticam 1300 imaging system (Motic China Group Co. Ltd. Xiamen, China). All measurements were made with the ruler tool in Photoimpact 6.0 ESD extended (Ulead Systems, Inc., Taipei, Tw).

\section{DNA extraction, PCR amplification and nucleotide sequencing}

The extracted DNA was stored at $-20^{\circ} \mathrm{C}$. Taq enzyme and dNTP were from Shanghai Tiangen. DNA extraction was according to Liang et al. (2011). Amplification of Beta tubulin (TUB) was performed with Bt2a (5'-GGTAACCAAATCGGTGCTGCTTTC-3') and Bt2b (5'-ACCCTCAGTGTAGTGACCCTTGGC3') (Glass \& Donaldson 1995). RNA polymerase II largest subunit (RPB1) was with the primer CRPB1A (5'-CAYCCWGGYTTYATCAAGAA-3') and RPB1Cr (5'-CCNGCDATNTCRTTRTCCATRTA-3') (Castlebury et al. 2004). For the amplification of RNA polymerase II subunit two (RPB2) the forward primer 5'-GACGACCGTG ATCACTTTGG-3' and the reverse primer 5'-CCCATGGCCTGTTTGCCCAT-3' were used (Houbraken et al. 2007), and translation elongation factor 1 alpha (EF1a) was amplified with forward primer 5'-GCCCCCGGCCATCGTGACTTCAT-3' and reverse primer 5'-ATGACACCGACAGCGACGGT 
CTG-3'(Houbraken et al. 2007). The PCR products were purified using the UNIQ-10 column PCR Products Purification kit (no. SK1141; Sangon Biotech (Shanghai) Co. Ltd', Shanghai, China) according to the manufacturer's protocol and sequenced with the above primers at Sangon Biotech (Shanghai) Co. Ltd.

\section{Phylogenetic analysis}

DNA sequences generated in this study were assembled and edited using Lasergene software (version 6.0, DNASTAR). Sequences of EF1a, RPB1, RPB2 and TUB from 33 taxa (with Nomuraea atypicola as an outgroup) based on the BLAST sequence similarity to our sample and the literature (Kepler et al. 2014) were downloaded from GenBank. Multiple sequence alignments for EF1a, RPB1, RPB2 and TUB were constructed with MAFFT v7.037b (Katoh et al. 2013). Sequences alignments were manually edited with MEGA v. 6 (Tamura et al. 2013). A concatenated dataset (EF1a+RPB1+RPB2+TUB) was assembled using SequenceMatrix v. 1.7.8 (Vaidya et al. 2011). Concordance between genes was assessed with the 'hompart' command of PAUP4.0b10 (Swofford 2002).

A phylogenetic tree was inferred from the combined four-locus dataset with MrBayes 3.2 (Ronquist et al. 2012). Two runs were executed simultaneously for 10000000 generations, saving trees every 500 generations. The SYM+G nucleotide substitution model and GTRGAMMA rate variation model were used for all partitions. All phylogenetic reconstructions were performed with services available from the CIPRES web portal (Miller et al. 2010). Our aligned EF1a+RPB1+RPB2+TUB dataset is 3,982 bp long, and is available in TreeBASE under submission ID 19899.

\section{Results}

\section{Phylogenetic analyses}

Newly generated EF1a, RPB1, RPB2 and TUB sequences of GZAC IFR1006 were deposited in GenBank (KT166031, KT961694, KT166032, and KT166033, respectively). M. dendrolimatilis assorts to a single clade by itself, supported by a high posterior probability in our maximum likelihood and Bayesian analysis (Fig. 1).

Metarhizium dendrolimatilis Z.Q. Liang, W.H. Chen, Y.F. Han \& D.C. Jin, sp. nov.

Fig. 2 MycoBank 812866

Facesoffungi number: FoF 02608

Etymology - named for its host Dendrolimus sp..

On Dendrolimus sp. caterpillar, $2.5 \mathrm{~cm}$ long, $0.9 \mathrm{~cm}$ wide. Asexual morph: Synnemata are scattered, white to earth yellow at the base, white to green at the upper edges. The spore mass, when present, is $0.6-1.2 \mathrm{~cm}$ long, $282-333 \mu \mathrm{m}$ wide. Conidiophores arise from the lateral hyphae of the synnemata, bearing loosely verticillate, with each branch bearing 2-10 phialides; the verticillate space is $22.5-35 \mu \mathrm{m}$. Phialides are closely appressed, rarely solitary, and directly on the conidiophores, with base globes, and a short neck, 2.2-3.2 $\mu \mathrm{m}$. Conidia are one-celled, smoothwalled, ellipsoid, $3.2-5.4 \times 2.2-3.2 \mu \mathrm{m}$, or globose, $2.2-4.3 \mu \mathrm{m}$ long.

Colonies grow moderately fast on PDA at $25^{\circ} \mathrm{C}$; after 14 days, 20.5-24.5 mm in diameter, velutinous, light yellow green, with white floccose margin. The reverse is light yellow. Hyphae are septate, smooth-walled, hyaline, and 1.5-1.8 $\mu \mathrm{m}$ wide. Conidiophores are hyaline, smooth walled, cylindrical, arise from aerial hyphae, and 9.3-15.0 $\times 1.2-3.4 \mu \mathrm{m}$. Phialides are in a cluster of two to five, arising on the lateral and end of conidiophores, subglobose at the base, and 4.3-5.4 $\mu \mathrm{m}$ long. Conidia are single or formed in long chains, one celled, hyaline, smooth walled, ellipsoid, and 3.8$10 \times 2-2.5 \mu \mathrm{m}$.

Colonies grow slowly on Czapek agar at $25^{\circ} \mathrm{C}$; after 14 days, $18.5-20.0 \mathrm{~mm}$ in diameter, thinly lawned, with an off white margin. Conidiophores 13.5-14.0 $\times 1.1-1.6 \mu \mathrm{m}$. Phialides 3.9-6.5 $\times 1.9-2.5 \mu \mathrm{m}$. Conidia 3.0-3.9 $\times 1.3-2.2 \mu \mathrm{m}$. Other characteristics were similar to those on PDA growth.

Sexual morph - Unknown 


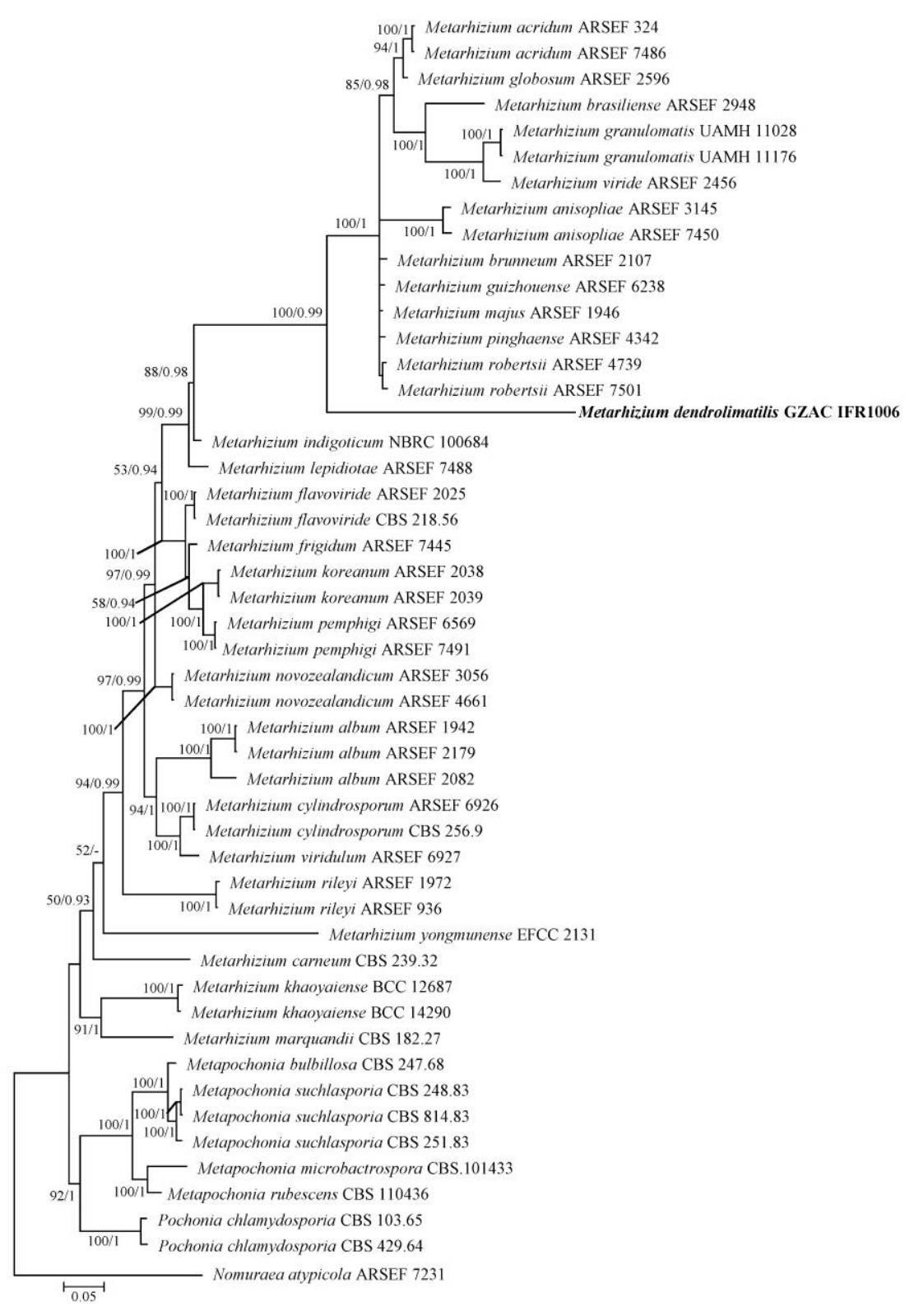

Fig. 1 - Phylogenetic tree generated from maximum parsimony and Bayesian method analysis based on EF1a+RPB1+RPB2+TUB sequences. Statistical support values of Maximum-parsimony bootstrap values $(\geqslant 50 \%)$ and Bayesian posterior probabilities $(\geqslant 0.9)$ are shown at nodes.

Type - China, Guizhou Province, Guiyang, Tongmuling, N26²3', E106 $40^{\prime}$, on the Dendrolimus sp. in the pinewood, 6 October 2013, W.H. Chen (HXDX.1006, holotype)

Known distribution - Guiyang and Qiandongnan Miao and Dong Autonomous Prefecture Guizhou Province, China

Material examined - China, Guizhou Province, Qiandongnan Miao and Dong Autonomous Prefecture, Cenggong County $\left(27^{\circ} 12^{\prime} \mathrm{N}, 108^{\circ} 84^{\prime} \mathrm{E}\right)$, on the Dendrolimus sp. in the pinewood, 24 October 2015, L.Q. Yu, paratype CC1024, ex-paratype culture GZAC JS102401).

Notes - We compared $M$. dendrolimatilis with similar species of this genus. Only one species has ellipsoidal conidia and synnemata, M. flavoviride var. minus Rombach, Humber \& D.W. Roberts, but $M$. dendrolimatilis is easily distinguishable from $M$. flavoviride var. minus by its globose phialides $(2.2-3.2 \mu \mathrm{m})$. 

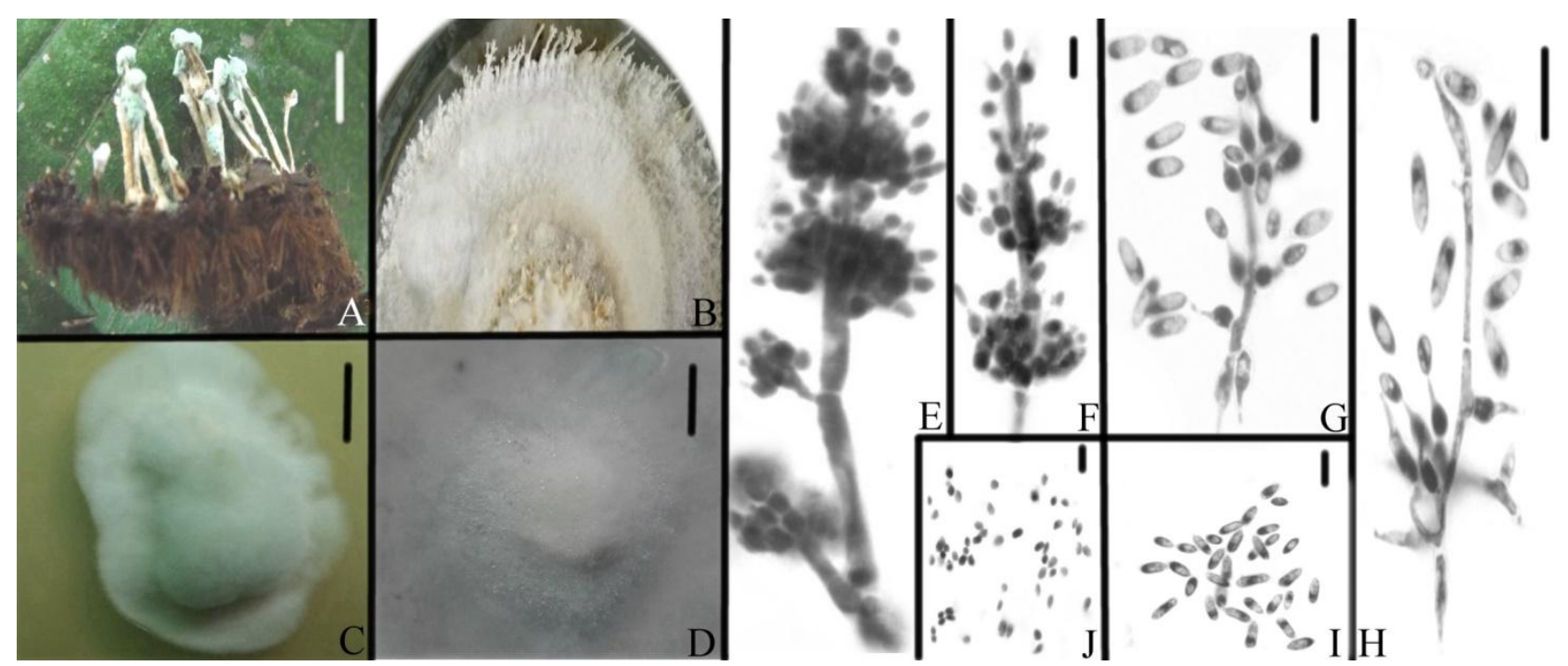

Fig. 2 - Metarhizium dendrolimatilis (HXDX.1006, holotype). A Synnemata on the Dendrolimus sp. B Synnemata on the edge of the PDA plate after $20 \mathrm{~d}$ at $25^{\circ} \mathbf{C}$. C, D. The colony on PDA and Czapek after $14 \mathrm{~d}$ at $25^{\circ} \mathrm{C}$. E, F, J. Conidiophores, phialides and conidia arising on the natural substrate. G, H, I. Conidiophores, phialides and conidia formed on PDA. - Bar: A, C, D = $0.5 \mathrm{~cm}$; E, F, G, H, I, J = $10 \mu \mathrm{m}$.

\section{Discussion}

As the originally described by Sorokin and subsequently emended (Rombach et al. 1987), the main taxonomic criteria for the genus Metarhizium are the shape of its phialides and conidia, with or without a sporulation zone in a hymenium-like layer, as well as a clustering behavior forming aprismatic spore masses. Conidia size has great significance in Metarhizium classification; the color of hyphae and conidia is of secondary significance. We compared $M$. dendrolimatilis with similar species of this genus. Only one species has ellipsoidal conidia and synnemata, $M$. flavoviride var. minus Rombach, Humber \& D.W. Roberts, but M. dendrolimatilis is easily distinguishable from $M$. flavoviride var. minus by its globose phialides $(2.2-3.2 \mu \mathrm{m})$.

The most complete taxonomic treatments of Metarhizium to date were conducted using a four-locus phylogeny (Kepler et al. 2014, Montalva et al. 2016). We decided to use the same fourlocus multigene phylogenetic approach in our present study. Most branches are strongly supported in the phylogenetic tree inferred from our Bayesian analysis. Strain GZAC IFR1006 assorts to a single clade alone, supporting its unique identification based on morphological characters. Metarhizium dendrolimatilis is a new species in the genus Metarhizium.

Despite our evidence, based on morphological characters and a phylogenetic analysis, which shows that Metarhizium dendrolimatilis is a new species, a crucial question remains: What is the molecular genetic background for the presence of synnemata in M. dendrolimatilis. Gao et al. (2011) presented a comparative analysis of $M$. anisopliae and $M$. acridum genome sequences, and speculates that the two species diverged from an ancestral plant endophytic fungi similar to Epichloe fetucae, or an ancestral wheat pathogenic fungi similar to Fusarium graminearum, ca. 3343 million years ago. Metarhizium's habitat changed from plant to soil during this divergence; a concurrent host shift occurred to soil inhabiting insects. Metarhizium taii Z.Q. Liang \& A.Y. Liu and M. majus (J.R. Johnst.) J.F. Bisch. (the anamorph of M. brittlebankisoides (Zuo Y. Liu, Z.Q. Liang, Whalley, Y.J. Yao \& A.Y. Liu) Kepler, S.A. Rehner \& Humber) (Liang et al. 1991, Liu et al. 2001) are two species that continued to adaptively radiate into different forest ecological conditions, and evolved different kinds of synnemata for different means of spore dispersal (Kepler 
et al. 2014). The formation of synnemata as a newly derived character in Metarhizium is a product of convergent evolution, and serves as an adaption for the dispersal of spores in very specific ecological conditions.

\section{Acknowledgements}

This work was financed by the National Natural Science Foundation of China (Grant No. 31093440, 31493010 \& 31493011, 31460010), the Special Fund of Excellent Youth Talents in Guizhou Province (Grant No. R2013-05), and the Provincial Outstanding Graduate Program for Agricultural Entomology and Pest Control (NO: Qianjiaoyanhe ZYRC (2013) 010).

\section{References}

Bischoff JF, Rehner SA, Humber RA. 2006 - Metarhizium frigidum sp. nov.: a cryptic species of M. anisopliae and a member of the M. flavoviride complex. Mycologia 98, 737-745.

Bischoff JF, Rehner SA, Humber RA. 2009 - A multilocus phylogeny of the Metarhizium anisopliae lineage. Mycologia 101, 512-530.

Castlebury LA, Rossman AY, Sung GH, Hyten AS, Spatafora JW. 2004 - Multigene phylogeny reveals new lineage for Stachybotrys chartarum, the indoor air fungus. Mycological Research 108, 864-872.

Curran J, Driver F, Ballard JWO, Milner RJ. 1994 - Phylogeny of Metarhizium: analysis of ribosomal DNA sequence data. Mycological Research 98, 547-552.

Driver F, Milner RJ, Trueman JW. 2000 - A taxonomic revision of Metarhizium based on a phylogenetic analysis of rDNA sequence data. Mycological Research 104, 134-150.

Gao Q, Jin K, Ying SH, Zhang YJ, Xiao GH, Shang YF, Duan ZB, Hu X, Xie XQ, Zhou G, Peng GX, Luo ZB, Huang W, Wang B, Fang WG, Wang SB, Zhong Y, Ma LJ, St Leger RJ, Zhao GP, Pei Y, Feng MG, Xia YX, Wang CS. 2011 - Genome sequencing and comparative transcriptomics of the model entomopathogenic fungi Metarhizium anisopliae and M. acridum. PLoS Genet 7, e1001264.

Glass NL, Donaldson GC. 1995 - Development of primer sets designed for use with the PCR to amplify conserved genes from filamentous ascomycetes. Applied and Environmental Microbiology 61, 1323-1330.

Houbraken J, Due M, Varga J, Meijer M, Frisvad JC, Samson RA. 2007 - Polyphasic taxonomy of Aspergillus section Usti. Studies in Mycology 59, 107-128.

Katoh K, Standley DM. 2013 - MAFFT multiple sequence alignment software version 7: improvements in performance and usability. Molecular Biology and Evolution 30, 772-780.

Kepler RM, Humber RA, Bischoff JF, Rehner SA. 2014 - Clarification of generic and species boundaries for Metarhizium and related fungi through multigene phylogenetics. Mycologia 106, 811-829

Latch GCM. 1965 - Metarhizium anisopliae (Metsch.) Sorok. strains in New Zealand and the possible use for controlling pasture inhabiting insects. New Zealand Journal of Agricultural Research 8, 384-396.

Liang JD, Han YF, Zhang JW, Du W, Liang ZQ, Li ZZ. 2011 - Optimal culture conditions for keratinase production by a novel thermophilic Myceliophthora thermophila strain GZUIFR-H49-1. Journal of Applied Microbiology 110, 871-880.

Liang ZQ, Liu AY, Liu JL. 1991 - A new species of the genus Cordyceps and its Metarhizium anamorph. Acta Mycologica Sinica 10, 257-262.

Liu ZY, Liang ZQ, Whalley AJS, Yao YJ, Liu AY. 2001 - Cordyceps brittlebankisoides, a new pathogen of grubs and its anamorph, Metarhizium anisopliae var. majus. Journal of Invertebrate Pathology 78, 178-182.

Maniania NK, Bugeme DM, Wekesa VW, Delalibera JrI, Knapp M. 2008 - Role of entomopathogenic fungi in the control of Tetranychus evansi and Tetranychus urticae (Acari: Tetranychidae), pests of horticultural crops. Experimental and Applied Acarology 46, 259-274.

Miller MA, Pfeiffer W, Schwartz T. 2010 - Creating the CIPRES Science Gateway for inference of large phylogenetic trees. In Gateway Computing Environments Workshop (GCE), 1-8.

Montalva C, Collier K, Rocha LF, Inglis PW, Lopes RB, Luz C, Humber RA. 2016 - A natural fungal infection of a sylvatic cockroach with Metarhizium blattodeae sp. nov., a member of the $M$. flavoviride species complex. Fungal Biology 120, 655-665. 
Petch T. 1931 - Notes on entomogenous fungi. Transactions of the British Mycological Society 16, 55-75.

Petch T. 1935 - Notes on entomogenous fungi. Transactions of the British Mycological Society 19, 161-194.

Rakotonirainy MS, Cariou ML, Brygoo Y, Riba G. 1994 - Phylogenetic relationships within the genus Metarhizium based on 28S rRNA sequences and isozyme comparison. Mycological Research 98, 225-230.

Roberts DW, St Leger RJ. 2004 - Metarhizium spp., cosmopolitan insect-pathogenic fungi: mycological aspects. Advances in Applied Microbiology 54, 1-70.

Rombach MC, Humber RA, Evans HC. 1987 - Metarhizium album, a fungal pathogen of leaf-and planthoppers of rice. Transactions of the British Mycological Society 88, 451-459.

Rombach MC, Humber RA, Roberts DW. 1986 - Metarhizium flavoviride var. minus, var. nov., a pathogen of plant-and leafhoppers on rice in the Philippines and Solomon Islands. Mycotaxon 27, 87-92.

Ronquist F, Teslenko M, van der Mark P, Ayres DL, Darling A, Höhna S, Larget B, Liu L, Suchard MA, Huelsenbeck JP. 2012 - MrBayes 3.2: efficient Bayesian phylogenetic inference and model choice across a large model space. Systematic Biology 61, 539-542.

Stafford KC, Allan SA. 2010 - Field applications of entomopathogenic fungi Beauveria bassiana and Metarhizium anisopliae F52 (Hypocreales: Clavicipitaceae) for the control of Ixodes scapularis (Acari: Ixodidae). Journal of Medical Entomology 47, 1107-1115.

Sung GH, Hywel-Jones NL, Sung JM, Luangsa-ard JJ, Shrestha B, Spatafora JW. 2007 - Phylogenetic classification of Cordyceps and the clavicipitaceous fungi. Studies in Mycology 57, 5-59.

Swofford DL. 2002 - PAUP* 4.0b10: phylogenetic analysis using parsimony (*and other methods). Sunderland, MA: Sinauer.

Tamura K, Stecher G, Peterson D, Filipski A, Kumar S. 2013 - MEGA6: molecular evolutionary genetics analysis version 6.0. Molecular Biology and Evolution http://dx.doi.org/10.1093/molbev/mst197.

Tulloch M. 1976 - The genus Metarhizium. Transactions of the British Mycological Society 66, 407-411.

Vaidya G, Lohman DJ, Meier R. 2011 - SequenceMatrix: concatenation software for the fast assembly of multigene datasets with character set and codon information. Cladistics 27, 171-180.

Wekesa VW, Knapp M, Maniania NK, Boga HI. 2006 - Effects of Beauveria bassiana and Metarhizium anisopliae on mortality, fecundity and egg fertility of Tetranychus evansi. Journal of Applied Entomology $130,155-159$.

Wekesa VW, Maniania NK, Knapp M, Boga HI. 2005 - Pathogenicity of Beauveria bassiana and Metarhizium anisopliae to the tobacco spider mite Tetranychus evansi. Experimental and Applied Acarology 36, 41-50. 